\title{
A negative premovement potential in the rat
}

\author{
FRANS J. VOORN \\ Tilburg University, Tilburg, The Netherlands
}

\begin{abstract}
Premovement potentials, which are known to be negative in humans and monkeys, were investigated in the rat. Five rats were trained to reach through a vertical slit with the right forepaw and to depress, for more than $200 \mathrm{msec}$, a lever located $15 \mathrm{~mm}$ behind the slit. Left forepaw position was controlled. Transcortical surface-to-depth EEG was recorded from the left and right sensorimotor forelimb areas. In 4 subjects, a negative-going premovement potential was reliably obtained in the averaged contralateral EEG. There was a more or less linear rise in negative potential from 200 msec before lever release until the period of maximal movement velocity, during which the potential turned positive. Ipsilateral premovement potentials were small or absent. No positive premovement potentials were found. It is suggested that the contralateral premovement potential is the rat homologue of the asymmetrical, late part of the Bereitschaftspotential found in humans and monkeys.
\end{abstract}

Since Kornhuber and Deecke (1965) described averaged, scalp-recorded movement-related potentials (MRPs) in humans, the cortical generators of several MRP components have been investigated in humans and monkeys. More than half a second before a voluntary movement of the hand, the electroencephalogram (EEG) recorded from the hand area of the contralateral motor cortex shows a slowly developing surface-negative potential (Bereitschaftspotential, or Nl). In scalp recordings, the slow negative potential begins to predominate above the contralateral motor cortex at $400 \mathrm{msec}$ prior to movement. Between 90 and $60 \mathrm{msec}$ before electromyogram (EMG) onset, this potential is followed by a sharp negative wave (motor potential, or $\mathrm{N} 2$ ) that reaches a maximum amplitude at 10 to $30 \mathrm{msec}$ after EMG onset. Occasionally, a small, positive deflection marks the beginning of N2 (Arezzo \& Vaughan, 1975; Deecke, Grözinger, \& Kornhuber, 1976). As far as the contralateral precentral motor cortex is concerned, investigations of possible sources of N1 and N2 have demonstrated (1) restriction of the maximum of the epidural amplitude distribution to the hand area in humans (Pieper, Goldring, Jenny, \& McMahon, 1980) and in monkeys (Arezzo \& Vaughan, 1975; Pieper et al., 1980), (2) transcortical polarity inversion of N1 and N2 in the hand area (Arezzo \& Vaughan, 1975; Hashimoto, Gemba, \& Sasaki, 1979; Pieper et al., 1980), and (3) an increase in multiple unit activity exclusively in the deep cortical layers of the hand area, concurrent with N2 (Arezzo \& Vaughan, 1980; Arezzo, Vaughan, $\&$ Koss, 1977). These findings have established the selfinitiated contraction of hand muscles in humans and monkeys as a sufficient condition for the occurrence of $\mathrm{N} 1$

The author gratefully acknowledges the advice of C. H. M. Brunia and P. F. M. Kop and the technical assistance of Hans Adamse. Special thanks for histological help are extended to A. H. M. Lohman, Free University, Amsterdam. Reprint requests should be addressed to: F. J. Voorn, Department of Psychology, Tilburg University, P.O. Box 90153 , 5000 LE Tilburg, The Netherlands. and N2 in the averaged EEG of the hand area of the contralateral motor cortex.

The modeling of these potentials in the lissencephalic brain of the rat would simplify the localization of neuronal generators. In addition, it could be useful in elucidating the causal significance of premovement potentials in a rat model of movement epilepsy. However, no negative cortical potentials preceding discrete, trained movements have been described in rats. On the contrary, the only premovement potential that has been reported in rats is a positive wave. Megirian, Burešová, Bureš, and Dimond (1974) recorded epidural EEG monopolarly above the sensorimotor cortex of rats reaching for food in a narrow tube. Interruption of a light beam crossing the tube served as the trigger signal for the averaging process. The contralateral MRP consisted of a negative potential at $28 \mathrm{msec}$ after, and a less regular positive wave at $36 \mathrm{msec}$ before, the trigger signal. However, the starting position of the forepaw was not controlled. Therefore, the trigger signal followed EMG onset by a variable delay that may be estimated on the basis of the following facts: (1) modal reaching behavior consisted of a cyclic motor pattern of five reaches per second, (2) the light beam was located at two-thirds of the way along the modal paw trajectory, and (3) modal beam interruption lasted $100 \mathrm{msec}$ (Megirian et al.). Consequently, the beam remained uninterrupted for $100 \mathrm{msec}$ on the average, leaving some $50 \mathrm{msec}$ between the start of the reaching movement and the trigger moment. Therefore, whether the positive pretrigger wave found can properly be called a premovement potential is questionable. It may, in fact, be the homologue of the first positive postmovement potential found in monkeys, which is, in part, a reflection of shortlatency reafferent activity (Arezzo \& Vaughan, 1980). The latency of the first positive component of the cortical response to electrical stimulation of contralateral forepaw muscle in the anesthetized rat is 5 to $7 \mathrm{msec}$ (Angel \& Lemon, 1975). Therefore, a positive reafferent potential may be expected in the rat MRP within $10 \mathrm{msec}$ after 
EMG onset. Such an early positive postmovement potential may have occurred well before the trigger moment in Megirian et al.'s (1974) study.

The present study of rat MRPs was designed to reduce uncertainty about limb position prior to the trigger moment. This was achieved by training the animals to hold a lever down with the right forepaw for at least $200 \mathrm{msec}$. Concurrent left forepaw position was controlled. The transcortical EEG of the left and right sensorimotor areas was averaged with respect to lever release.

\section{METHOD}

\section{Subjects and Apparatus}

Five male Wistar rats were maintained at $85 \%$ of their free-feeding body weights on a 23-h food-deprivation schedule. Experimentation took place in the 12-h light phase of a 24-h light-dark cycle.

A standard rodent Skinner box (BRS/LVE 143-21) was used for the conditioning procedure. The front wall of the box contained a response lever, a $12-\mathrm{mm}$-wide white cue light at $50 \mathrm{~mm}$ above the middle of the lever, and a feeder to deliver $45-\mathrm{mg}$ food pellets. The lever protruded $22 \mathrm{~mm}$ from the wall at $50 \mathrm{~mm}$ above floor level, and activated a microswitch when pressed downward with a force of $0.25 \mathrm{~N}$. Maximal vertical lever displacement was $2 \mathrm{~mm}$. Behind the wall, a small rod extended from the lever into the beam of a photoelectric device in order to produce an electrical analogue of lever displacement. This signal will be referred to henceforth as the "mechanogram." All bodily contacts with the lever, except from the forepaw, were obstructed by a $70-\mathrm{mm}$-wide metal shield that was placed parallel to, and $37 \mathrm{~mm}$ in front of, the wall. Its vertical axis was symmetrical with respect to the lever. In the middle of the shield, an 8-mm-wide vertical slit extended from 25 to $90 \mathrm{~mm}$ above floor level. From the lower left corner of the shield, a $25 \times 25 \mathrm{~mm}$ pedal protruded horizontally near floor level. Its right edge was $10 \mathrm{~mm}$ from the middle of the shield. The axles of the lever and floor pedal were parallel to both the floor and the shield. General illumination was provided by a houselight near the ceiling. The experimental cage was placed in a sound-attenuating cubicle and extraneous sounds were masked by white noise.

Bipolar electrodes were made by gluing together two $0.25-\mathrm{mm}$ wide stainless steel wires and soldering them to Amphenol connectors (2741-000). The distance between the two uninsulated tips was $1.5 \mathrm{~mm}$. EEGs were recorded on a Beckman R-612 instrument. Bandpass cutoff frequencies (at $-3 \mathrm{~dB}$ ) were 0.16 and $28 \mathrm{~Hz}$ (rolloff $6 \mathrm{~dB}$ /octave). The EEG signals, along with the mechanogram and a code signal reflecting aspects of the animal's behavior, were stored on magnetic tape to be digitized and analyzed off-line

\section{Procedure}

Using standard operant conditioning methods, the subjects were trained to reach through the slit with the right forepaw and to depress the lever while holding the floor pedal down with the left forepaw. The cue light was introduced to separate the left- and right-forepaw movements temporally. When the floor pedal was held down for $1 \mathrm{sec}$, the cue light came on. It remained on only as long as the floor pedal was held down. The beginning and end of a lever response were defined by closure and deactivation of the microswitch, respectively. The only lever responses to be reinforced (with a conditioned reinforcer) were those beginning at least $300 \mathrm{msec}$ after cue onset and ending at least $200 \mathrm{msec}$ later, while the floor pedal was still held down. These responses were reinforced by turning off the houselight and the noise for 400 msec immediately following lever release. This 400 -msec period was followed by food-pellet delivery according to a fixed-ratio schedule. The ratio was 10 or less, depending on the subject's proficiency. Houselight, noise, food delivery, cue light, and pedal and lever depressions were encoded in the behavior-code signal.

After 90 training sessions, the animals were subjected to stereotaxic surgery under chloralhydrate $(300 \mathrm{mg} / \mathrm{kg}$ i.p. $)$ anesthesia. Two bipolar electrodes were implanted $2.5 \mathrm{~mm}$ to the left and right of the sagittal plane, $1.5 \mathrm{~mm}$ in front of bregma. These coordinates give the approximate location of the cortical motor areas of the forelimb (Elger, Speckmann, Caspers, \& Janzen, 1977; Hall \& Lindholm, 1974; Neafsey \& Sievert, 1982). At each side, the bipolar electrode was lowered until its upper tip reached the dura. A stainless steel screw for grounding was driven into the skull $7 \mathrm{~mm}$ anterior and $1 \mathrm{~mm}$ to the right of bregma. The electrode assembly was fixed to the skull with dental cement.

Starting on Day 4 after surgery, EEGs were recorded during four experimental sessions for each subject. The intervals between sessions were 3 or 4 days. In Subject C3, EEG recording had to be postponed until the fourth postoperative week due to technical difficulties. Differential EEG signals were obtained bilaterally from transcortical surface-to-depth derivations in order to selectively record potentials generated by radially oriented dipoles in the motor area. By virtue of the small tip separation, this derivation is relatively insensitive to distant potential generators.

After completion of the experiment, the brains were prepared for histological examination. Following electrolytical iron deposition at the lower electrode tips, the brain was perfused with a mixture of formalin and potassium ferrocyanide. Coronal sections were stained with cresyl-violet and examined using Zilles's delineations of cortical areas (Zilles, 1985; Zilles \& Wree, 1985).

\section{Data Analysis}

Analog-to-digital conversion at a rate of $150 \mathrm{samples} / \mathrm{sec}$ was accomplished simultaneously for the EEG signals from the left and right sensorimotor cortices, the mechanogram, and the behaviorcode signal. The mechanogram served to trigger a routine that, for each signal, stored the samples of the 200 -msec intervals preceding and following the trigger moment. The resulting set of four 400 msec sample records, corresponding to one response, will be referred to as a "trial." The triggering occurred at the moment that the mechanogram reached the amplitude corresponding to deactivation of the lever switch. Consequently, the onset of lever release was recorded, but its temporal relationship to the trigger moment depended upon the subject's behavior, brisk release movements being more tightly time-locked to the trigger moment than slack ones. The peak-to-peak amplitude of each EEG sample record guided the visual inspection of trials and their eventual removal in case of artifact.

In the last analytical stage before averaging, the remaining trials were sorted according to the behavior-code signal. Trials were removed if the floor pedal was released less than $100 \mathrm{msec}$ after lever release. Response classes considered for averaging were (1) reinforced responses, and (2) responses that occurred in the presence of the cue light but did not meet the temporal criteria for response duration or latency (henceforth to be called "unreinforced responses"'). By means of the mechanogram, trials could be allocated to subclasses that were relatively homogeneous with respect to stability of holding and briskness of movement. The same mechanogram criteria were applied as much as possible to reinforced and unreinforced responses of all four sessions of a subject. If more than $\mathbf{5 0}$ trials met the criteria, only $\mathbf{5 0}$ were randomly selected for averaging.

\section{RESULTS}

Session averages of both reinforced and unreinforced responses are shown for each subject in Figure 1. Since reinforcement implied a stimulus change at the trigger mo- 


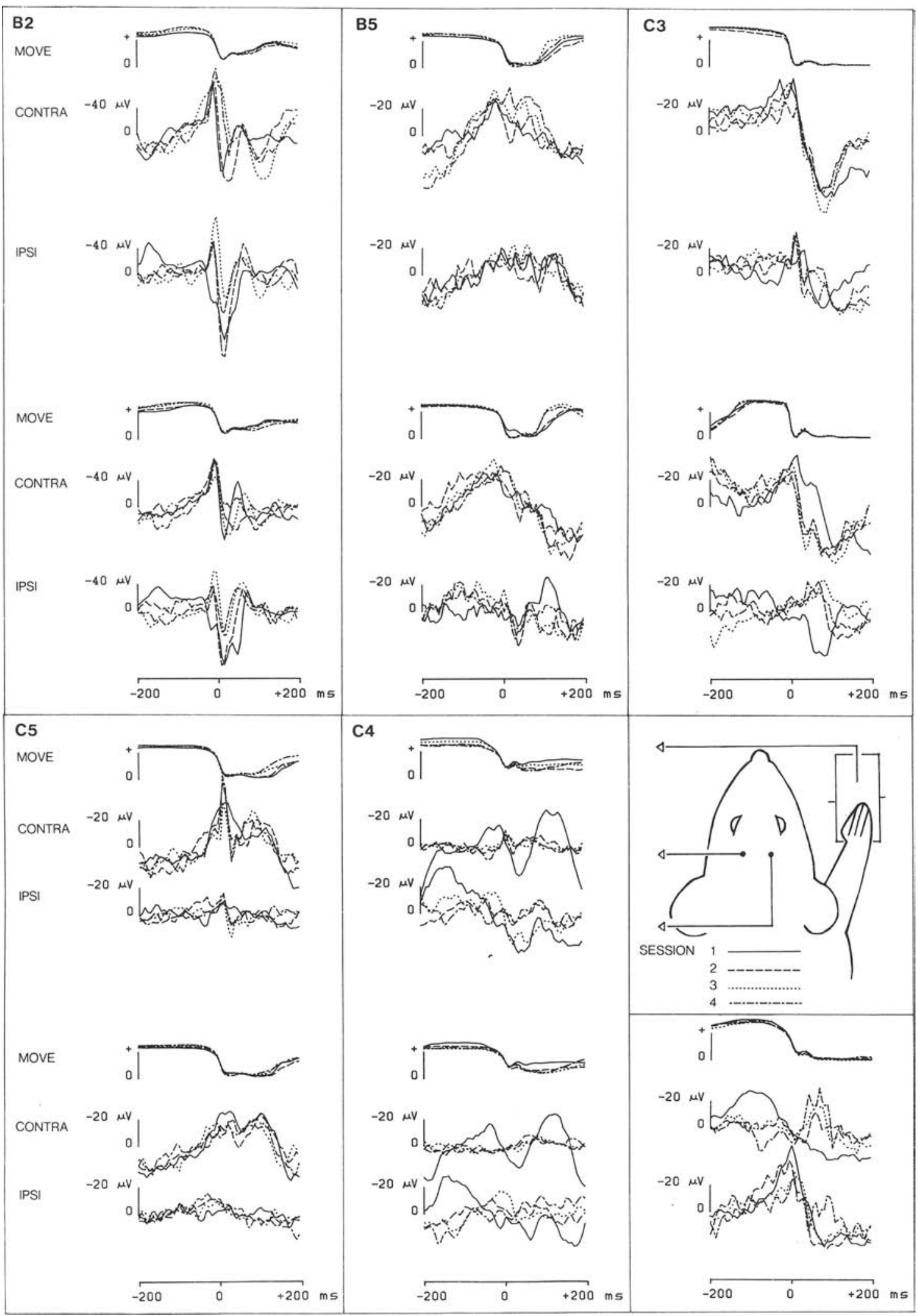

Figure 1. Averages of contralateral (second traces) and ipsilteral (third traces) potentials related to forepaw movement (first traces) are superimposed for Sessions 1 to 4 in the upper part (reinforced trials) and lower part (unreinforced trials) of each subject's panel. Averages comprise 50 trials. The bottom-right panel shows the averages of trials in which Subject $\mathrm{C4}$ did not hold the floor pedal down. 
ment, the averages of reinforced and unreinforced trials were compared. Noticeable differences in the posttrigger interval were found only in the contralateral EEG of Subjects $\mathrm{C} 4$ and $\mathrm{C} 5$ and in the ipsilateral EEG of Subjects C3 and C5. It was only in reinforced trials of Sessions 2,3 , and 4 that these EEGs showed a negative spike, extending from 0 to $40 \mathrm{msec}$ after the trigger moment (see Figure 1). Inspection of individual trials led to the conclusion that these sharp waves were artifacts caused by turning off the houselight and the noise. Apart from these few posttrigger differences, the averages of reinforced and unreinforced trials were comparable within each session and stable from session to session in 4 of the 5 subjects. The averages of these 4 subjects (B2, B5, C3, and C5) will be described first.

Generally, in the EEG contralateral to the moving paw, a negative-going premovement potential develops steadily until it reaches a maximum amplitude between -25 and $+35 \mathrm{msec}$ (see Figure 1). This potential starts at least $200 \mathrm{msec}$ prior to movement, except in the unreinforced trials of Subject C3. In this subject, unreinforced responses were generally shorter than $200 \mathrm{msec}$. The average of these responses shows that the contralateral negative premovement wave begins only after the downward movement of the lever has come to a halt. Only in Subject $\mathrm{B} 2$ is a sharp acceleration of the negative potential observed at -35 msec, when the averaged mechanogram indicates the beginning of movement. No reproducible positive potentials preceding the trigger moment have been observed. Immediately after the trigger moment, the contralateral EEG shows a positive-negative-positive wave pattern; such a pattern is, however, not regularly obtained in Subjects B5 and C3.

As compared with the contralateral EEG, ipsilateral averages were variable. When ipsilateral premovement potentials were present, they were slow negative waves with relatively small amplitudes. These potentials were not reliably obtained across reinforced and unreinforced trials or in all sessions of a subject. In Subject B2, a reproducible ipsilateral negative-positive-negative complex began at $-35 \mathrm{msec}$, just after the onset of movement, judging from the mechanogram.

In the fifth subject (C4), a negative-going premovement potential in the left hemisphere was obtained only on Postoperative Day 4 (Session 1). From Day 7 on, no systematic left-hemisphere potential could be found, whereas in the right-hemisphere average of Days 7 and 11 (Sessions 2 and 3), a negative maximum could be discerned before the trigger moment. This potential might be due to responses with the left forepaw. Pressing the lever with the left forepaw while holding the floor pedal down with the right forepaw or a hind paw would entail awkward postures, but it was not impossible. A reason to suspect left-paw lever responses in this subject was the appearance, from Day 7 on, of epileptiform spikes in the EEG. By Day 14 (Session 4), these spikes seemed responsebound, often occurring in the left-hemisphere EEG 50 to $100 \mathrm{msec}$ after the trigger moment. (Trials with spikes were discarded from the averages.) In unilateral reaching tasks, a switch to heterolateral reaching is promoted by small cortical lesions (Peterson \& Devine, 1963) and by an epileptic focus (unpublished observations) in the forepaw motor area. If the epileptic condition of Subject $\mathrm{C} 4$ were to increase the probability of left-paw lever responses, the category of responses without concurrent floor-pedal depression would be the one most likely to consist predominantly of left-paw responses. Therefore, trials of this category $(44,31,29$, and 27 trials for Sessions 1 to 4, respectively) were averaged for Subject C4. The bottom-right panel of Figure 1 shows that these averages consistently revealed a negative wave in the right hemisphere preceding the trigger moment. For the other subjects, averages of trials without floor-pedal depression did not show any reproducible potential variation in the right-hemisphere EEG.

Histological examination showed that all electrodes were situated in primary motor cortex on the rostral side of the anterior border of the forelimb area of SmI. The lower tips of all but three electrodes were located between pyramidal layer $\mathrm{V}$ and the corpus callosum. The left electrode of Subject C3 failed to reach the inner pyramidal layer. The lower electrode tips of Subject $\mathrm{C} 4$ penetrated the corpus callosum. In this subject, column-shaped islets of intensely stained cells, extending radially from the corpus callosum to the cortical surface, were found in the forelimb area of both hemispheres, 0.5-1.0 mm posterior to the electrodes.

\section{DISCUSSION}

In the present experiment, a negative-going premovement potential was reliably found in the transcortical surface-to-depth EEG of the contralateral sensorimotor area in 4 of 5 rats. The potential developed steadily from at least $200 \mathrm{msec}$ before the trigger moment until the period of maximal movement velocity, during which the potential turned positive. Ipsilateral premovement potentials were absent in some subjects and not reliably obtained in others.

The supposition that left-paw responses had entered into the averages of the fifth subject (C4) was originally based on movement-related epileptiform activity in the EEG. The histological abnormalities found in the forelimb area of Subject C4 support this supposition. It is argued, therefore, that some paradoxical averages of this subject are due to left-paw responses, and are, consequently, in line with the results of the other subjects. The additional analyses of trials without floor-pedal depression support this conclusion.

The slow, contralateral premovement potential was found principally in only one of two derivations, and its characteristic features recurred invariably from session to session, in reinforced as well as unreinforced responses, and across subjects. Therefore, an interpretation in terms of movement artifact is unlikely to be valid. Neither can the tonic activity of the right forepaw explain the differ- 
ence between the two EEG channels, since the left forepaw was simultaneously engaged in holding the floor pedal down.

In none of the subjects was a positive premovement wave obtained. However, a contralateral positivenegative-positive complex immediately followed the trigger moment in most subjects. This supports the supposition-as argued in the introduction-that the positive pretrigger potential described by Megirian et al. (1974) must be considered a postmovement potential.

In the motoneuron pool of the distal forepaw muscles of the rat, nearly half of the motoneurons mediate a monosynaptic connection between motor cortex and muscle (Elger et al., 1977). Correspondingly, EMG activity can occur as early as $2.7 \mathrm{msec}$ after cortical stimulation (Elger et al., 1977). This may serve to tightly lock cortical premovement potentials to the onset of movement. Yet, by the same token, the latest premovement potentials are bound to blur in averaging if there is a little play in the time-lock between the onset of movement and the trigger moment. In the present experiment, some temporal variation remained between mechanograms of different trials, even after classification. This could explain why a phasic, negative wave resembling $\mathrm{N} 2$ was absent in most subjects.

In the ipsilateral EEG, premovement potentials were either absent or similar to-but smaller than-contralateral ones and not reliably obtained in all averages of a subject. Such inconsistency is not uncommon in ipsilateral averages. In the monkey, an ipsilateral N1 is either absent (Arezzo \& Vaughan, 1975) or irregularly present, relatively small, and without transcortical polarity inversion (Gemba, Sasaki, \& Hashimoto, 1980; Hashimoto et al., 1979, 1981). In humans, ipsilateral premovement potentials are not present in epidural recordings (Pieper et al., 1980). The finding of a small, ipsilateral N2 in the monkey (Arezzo \& Vaughan, 1975) is compatible with activity increases in precentral pyramidal tract neurons preceding visually triggered movement (Evarts, 1966). In rats, a direct corticospinal projection to ipsilateral motoneurons of distal forepaw muscles exists (Elger et al., 1977). It may have been involved in the movement. However, adventitious reinforcement of concurrent activity in the arm contralateral to the explicitly reinforced movement can maintain such untoward activity. This may explain some ipsilateral inconsistencies in the present data and in the literature.

The present finding of an almost exclusively contralateral, slow, negative premovement wave runs parallel to observations in humans (Pieper et al., 1980) and monkeys (Arezzo \& Vaughan, 1975). Our results suggest that the negative premovement wave is the rat homologue of the contralaterally predominating part of the Bereitschaftspotential found in humans and monkeys. It supposedly reflects preliminary excitation of the apical dendrites of pyramidal tract neurons involved in the movement (Deecke et al., 1976; Hashimoto et al., 1979).

\section{REFERENCES}

ANgel, A., Lemon, R. N. (1975). Sensorimotor cortical representation in the rat and the role of the cortex in the production of sensory myoclonic jerks. Journal of Physiology, 248, 465-488.

Arezzo, J., \& Vaughan, H. G., JR. (1975). Cortical potentials associated with voluntary movements in the monkey. Brain Research, 88, 99-104.

AreZzo, J., \& Vaughan, H. G., Jr. (1980). Intracortical sources and surface topography of the motor potential and somatosensory evoked potential in the monkey. In H. H. Kornhuber \& L. Deecke (Eds.), Progress in brain research: Vol. 54. Motivation, motor and sensory processes of the brain: Electrical potentials, behaviour and clinicial use (pp. 77-83). Amsterdam: Elsevier.

Arezzo, J., Vaughan, H. G., JR., \& Koss, B. (1977). Relationship of neuronal activity to gross movement-related potentials in monkey pre- and postcentral cortex. Brain Research, 132, 362-369.

DeEcke, L., Grözinger, B., \& Kornhuber, H. H. (1976). Voluntary finger movement in man: Cerebral potentials and theory. Biological Cybernetics, 23, 99-119.

Elger, C. E., Speckmann, E.-J., Caspers, H., \& Janzen, R. W. C. (1977). Cortico-spinal connections in the rat. I. Monosynaptic and polysynaptic responses of cervical motoneurons to epicortical stimulation. Experimental Brain Research, 28, 385-404.

EvarTs, E. V. (1966). Pyramidal tract activity associated with a conditioned hand movement in the monkey. Journal of Neurophysiology, 29, 1011-1027.

Gemba, H., Sasaki, K., \& Hashimoto, S. (1980). Distribution of premovement slow cortical potentials associated with self-paced hand movements in monkeys. Neuroscience Letters, 20, 159-163.

HALL, R. D., \& Lindholm, E. P. (1974). Organization of motor and somatosensory neocortex in the albino rat. Brain Research, 66, 23-38.

Hashimoto, S., Gemba, H., \& SaSaki, K. (1979). Analysis of slow cortical potentials preceding self-paced hand movements in the monkey. Experimental Neurology, 65, 218-229.

Hashimoto, S., Gemba, H., \& SASAKI, K. (1981). Distribution of slow cortical potentials preceding self-paced hand and hindlimb movements in the premotor and motor areas of monkeys. Brain Research, 224, 247-259.

Kornhuber, H. H., \& DeECKe, L. (1965). Hirnpotentialänderungen bei Willkürbewegungen und passiven Bewegungen des Menschen: Bereitschaftspotential und reafferente Potentiale. Pflügers Archiv für die Gesamte Physiologie, 284, 1-17.

Megirian, D., Burešová, O., Bureš, J., \& Dimond, S. (1974). Electrophysiological correlates of discrete forelimb movements in rats. Electroencephalography \& Clinical Neurophysiology, 36, 131-139.

NeAfSEY, E. J., \& SiEverT, C. (1982). A second forelimb motor area exists in rat frontal cortex. Brain Research, 232, 151-156.

Peterson, G. M., \& Devine, J. V. (1963). Transfers in handedness in the rat resulting from small cortical lesions after limited forced practice. Journal of Comparative \& Physiological Psychology, 56, 752-756.

Pieper, C. F., Goldring, S., Jenny, A. B., \& McMahon, J. P. (1980). Comparative study of cerebral cortical potentials associated with voluntary movements in monkey and man. Electroencephalography \& Clinical Neurophysiology, 48, 266-292.

ZILLES, K. (1985). The cortex of the rat: A stereotaxic atlas. Berlin: Springer.

ZILLES, K., \& WreE, A. (1985). Cortex: Areal and laminar structure. In G. Paxinos (Ed.), The rat nervous system: Vol. 1. Forebrain and midbrain (pp. 375-415). Sydney: Academic Press.

(Manuscript received July 13, 1987; revision accepted for publication December 21, 1987.) 